\title{
Eighteen Primes in Arithmetic Progression
}

\author{
By Paul A. Pritchard
}

\begin{abstract}
There is an arithmetic progression of 18 primes, viz. $107928278317+k \cdot 9922782870$,
\end{abstract} $k=0,1, \ldots, 17$.

A long standing conjecture of Hardy and Littlewood [1] - the "prime $k$-tuples conjecture" - is that if $a_{1}, \ldots, a_{k}$ do not form a complete residue system (mod $p$ ) for any prime $p$, then there are infinitely many values of $x$ such that $x+a_{1}, \ldots, x+a_{k}$ are all prime. An interesting special case of this conjecture is that for all $n$ there are infinitely many arithmetic progressions of $n$ primes. Erdös has a beautiful (but hopeless) conjecture that would imply this:

If $\left\{a_{i}\right\}$ is any infinite sequence of integers for which $\sum a_{i}^{-1}$ is divergent, then the sequence contains arbitrarily long arithmetic progressions.

We have found an arithmetic progression of eighteen primes, breaking the previous record of seventeen due to Weintraub [3]. The progression is

$$
107928278317+k \cdot 9922782870, \quad k=0,1, \ldots, 17 .
$$

The prime factorization of the common difference is

$$
2 \cdot 3^{2} \cdot 5 \cdot 7 \cdot 11^{2} \cdot 13 \cdot 17 \cdot 19 \cdot 31
$$

As of the time of writing, this is the only known arithmetic progression of eighteen primes. It was discovered surprisingly early on in a search that is still in progress, having thus far run some 3500 hours on a Digital Equipment Corporation VAX-11/780. The search is over all arithmetic progressions with common difference a multiple of 9699690 - the product of the primes $\leqslant 19$ - and no term exceeding $30001 \cdot 9699690$, and is about $25 \%$ completed. It is likely that there are arithmetic progressions of eighteen primes with smaller last terms, because the factor of 19 in the common difference is unnecessary.

A complete report of all arithmetic progressions of at least seventeen primes that are found by our search will be prepared when the search is completed. The discovery was made using computer programs whose development is described at length in [2].

Department of Computer Science

Cornell University

Ithaca, New York 14853

1. G. H. HaRdy \& J. E. LitTLEwood, "Some problems of 'partitio numerorum' III: on the expression of a number as a sum of primes," Acta Math., v. 44, 1923, pp. 1-70.

2. P. Pritchard, "A case study of number-theoretic computation: searching for primes in arithmetic progression,” Sci. Comput. Programming. (To appear.)

3. S. Weintraub, "Seventeen primes in arithmetic progression," Math. Comp., v. 31, 1977, p. 1030.

Received November 8, 1982.

1980 Mathematics Subject Classification. Primary 10-04; Secondarv 10L20

(C)1983 American Mathematical Society $0025-5718 / 83 \$ 1.00+\$ .25$ per page 